Preprint typeset in JHEP style - HYPER VERSION

MIT-CTP-3595

\title{
From Entropy and Jet Quenching to Deconfinement?
}

\author{
Berndt Müller ${ }^{\dagger}$ and Krishna Rajagopal\# \\ ${ }^{\dagger}$ Department of Physics, Duke University, Durham, NC 27708-0305 \\ mueller@phy.duke.edu \\ \# Center for Theoretical Physics, Massachusetts Institute of Technology, \\ Cambridge, MA 02139 \\ krishna@ctp.mit.edu
}

\begin{abstract}
The challenge of demonstrating that the matter produced in heavy ion collisions is a deconfined quark-gluon plasma, as predicted by lattice QCD calculations, is the challenge of measuring the number of thermodynamic degrees of freedom $\nu \sim \varepsilon / T^{4}$ at the time $t_{0}$ at which the matter comes into approximate local thermal equilibrium and begins to behave like a hydrodynamic fluid. Data from experiments done at the Relativistic Heavy Ion Collider have been used to estimate $t_{0}$ and to put a lower bound on the energy density $\varepsilon\left(t_{0}\right)$. However, measuring $\nu$ has seemed out of reach, because no current data serve even as qualitative proxies for the temperature $T\left(t_{0}\right)$. We point out that $\nu$ may equally appropriately be defined via $\nu \sim s^{4} / \varepsilon^{3}$, where $s$ is the entropy density, which can be estimated from the measured final state entropy. This estimate is based on the testable assumption of an isentropic expansion. The observation of jet quenching has the potential to provide an upper bound on the energy density at early times. Our goal is to motivate such an analysis by pointing out that it would set a lower bound on $\nu$.
\end{abstract}

KeYwords: QCD, Heavy Ion Collisions, Jet Quenching, Deconfinement. 
Measurements of the final state produced in heavy ion collisions at $\sqrt{{ }^{s} \mathrm{NN}}=130$ and $200 \mathrm{GeV}$ at the Relativistic Heavy Ion Collider (RHIC) in Brookhaven have provided strong evidence that the matter created in these reactions is rapidly thermalized and has many properties expected from a strongly coupled quark-gluon plasma [1]. What is lacking, however, is direct evidence that could be linked to the prevalence of unconfined colored excitations, which are thought to be the defining feature of this novel state of matter.

At present, the best evidence for deconfinement of quarks may be that derived from the phenomenological success of valence quark recombination models [2], which are based on the assumption of the existence of a thermalized phase of unconfined "constituent quarks" immediately prior to hadronization. In particular, the quark recombination models explain the different saturation levels and thresholds for the elliptic flow of different hadrons in terms of a universal elliptic flow pattern at the valence quark level. A serious limitation of this evidence is that it addresses physics near the quark-hadron transition, which is inherently nonperturbative and thus not amenable to controlled theoretical approximations. It would be desirable to obtain evidence for the liberation of colored degrees of freedom in the matter created at RHIC which relates to thermalized matter present during an earlier stage of the reaction, when the temperature is higher, and to observables that allow for a more controlled theoretical description.

In QCD with quark masses as given in nature, there is no difference in symmetry between an equilibrated gas of hadrons and a quark-gluon plasma, implying that the transition between these two regimes of strongly interacting matter may be a continuous, albeit possibly rapid, crossover [3]. Indeed, this is what is predicted by ab initio calculations of QCD thermodynamics done using the methods of lattice gauge theory [4]. Hence, an experimental demonstration of deconfinement cannot be seen as the answer to some "yes/no" question, but must instead involve the measurement of some physical property of the matter created in heavy ion collisions that can also be predicted by controlled theoretical calculations, and which takes on quite different values below and above the crossover between a hadron gas and a quark-gluon plasma.

Because QCD is an asymptotically free theory, there are many quantities whose calculation is controlled at temperatures far above the crossover, where the effective QCD coupling $\alpha_{s}(T)$ becomes small. However, we do not expect that collisions at RHIC can create matter in this asymptotic regime. This means that in looking for quantities whose theoretical calculation is under control, we must ask what quantities can be calculated by rigorous numerical methods. Lattice calculations of non-thermodynamic quantities, like spectral functions [5] and viscosities [6], are still in their infancy and are presently restricted to QCD in the quenched approximation. In contrast, lattice calculations of QCD thermodynamics have reached maturity because they can be formulated conveniently in Euclidean quantum field theory, the natural arena for lattice QCD. Lattice simulations of the QCD equation of state including dynamical quarks of various numbers of flavors are available, which permit model-independent conclusions «].

In QCD thermodynamics, there is one observable whose value changes by more than an order of magnitude across the crossover transition from hadron gas to quark-gluon plasma, namely the effective number of thermodynamic degrees of freedom $\nu(T)$. A common defi- 
nition of $\nu(T)$ is via the relation

$$
\varepsilon(T)=\frac{\pi^{2}}{30} \nu(T) T^{4},
$$

where $\varepsilon$ and $T$ are the energy density and temperature, respectively. For an ideal gas of massless, noninteracting constituents, $\nu$ counts the number of bosonic degrees of freedom plus the number of fermionic degrees of freedom weighted by $7 / 8$. Eq. (1) makes the measurement of $\nu$ in heavy ion collisions seem a remote possibility, because there is nothing in the current suite of data from RHIC that is thought to serve as a proxy for the temperature at early times. The temperature at freezeout, when the matter is again hadronic, is well determined from many measurements of the hadronic final state. A measurement of the temperatures of the quark-gluon plasma presumed to be present at early times is one of the goals of studies of direct photon and dilepton emission in heavy ion collisions but, so far, no evidence for thermal photon or dilepton radiation has been observed at RHIC. Even if they will be observed eventually, thermal photons will only yield a weighted time-average of the temperature, which may not be sufficient to determine the function $\nu(T)$.

Here, we make the simple observation that $\nu$ can equally well be defined via the entropy density

$$
s(T)=\frac{2 \pi^{2}}{45} \nu(T) T^{3}
$$

and hence via

$$
\nu(T)=\frac{1215}{128 \pi^{2}} \frac{s^{4}}{\varepsilon^{3}}=0.96 \frac{s^{4}}{\varepsilon^{3}} .
$$

For an ideal gas of massless degrees of freedom, the expressions (17), (2) and (3) are equivalent definitions of $\nu$. We shall take (3) as our definition, because in so doing we realize that $\nu(T)$ can be measured without measuring $T$ itself. An ideal gas of ultrarelativistic pions has $\nu=3$, whereas an ideal gas of noninteracting gluons and three flavors of massless quarks has $\nu=47.5$.

Lattice QCD calculations show that $\nu$ increases by more than a factor of ten over a narrow range of temperatures centered at a crossover temperature $T_{c}=170 \pm 10 \mathrm{MeV}$, 4]. This rapid increase in the number of degrees of freedom is the direct consequence of deconfinement at high temperatures in QCD and, if we limit ourselves to thermodynamic observables, the measurement of $\nu$ is the only possible route to an experimental demonstration of deconfinement.

QCD does not describe an ideal gas of noninteracting quarks and gluons, except at infinite temperatures. And indeed, there is growing evidence from the RHIC experiments that the matter they are creating is strongly interacting, thermalizing rapidly, flowing like a liquid, and opaque to energetic partons produced within it [1]. In the case of strongly interacting matter, the different definitions of $\nu$ provided by (1), (2) and (3) are not equivalent, and furthermore $\nu$ cannot be considered as a count of well-defined degrees of freedom. Indeed, in an ideal liquid there are no well-defined long-lived quasiparticles. Nevertheless, upon choosing one definition - and we shall choose (3) - $\nu$ is reliably calculable on the lattice, potentially measurable as we shall discuss, and remains a valid measure of deconfinement. 


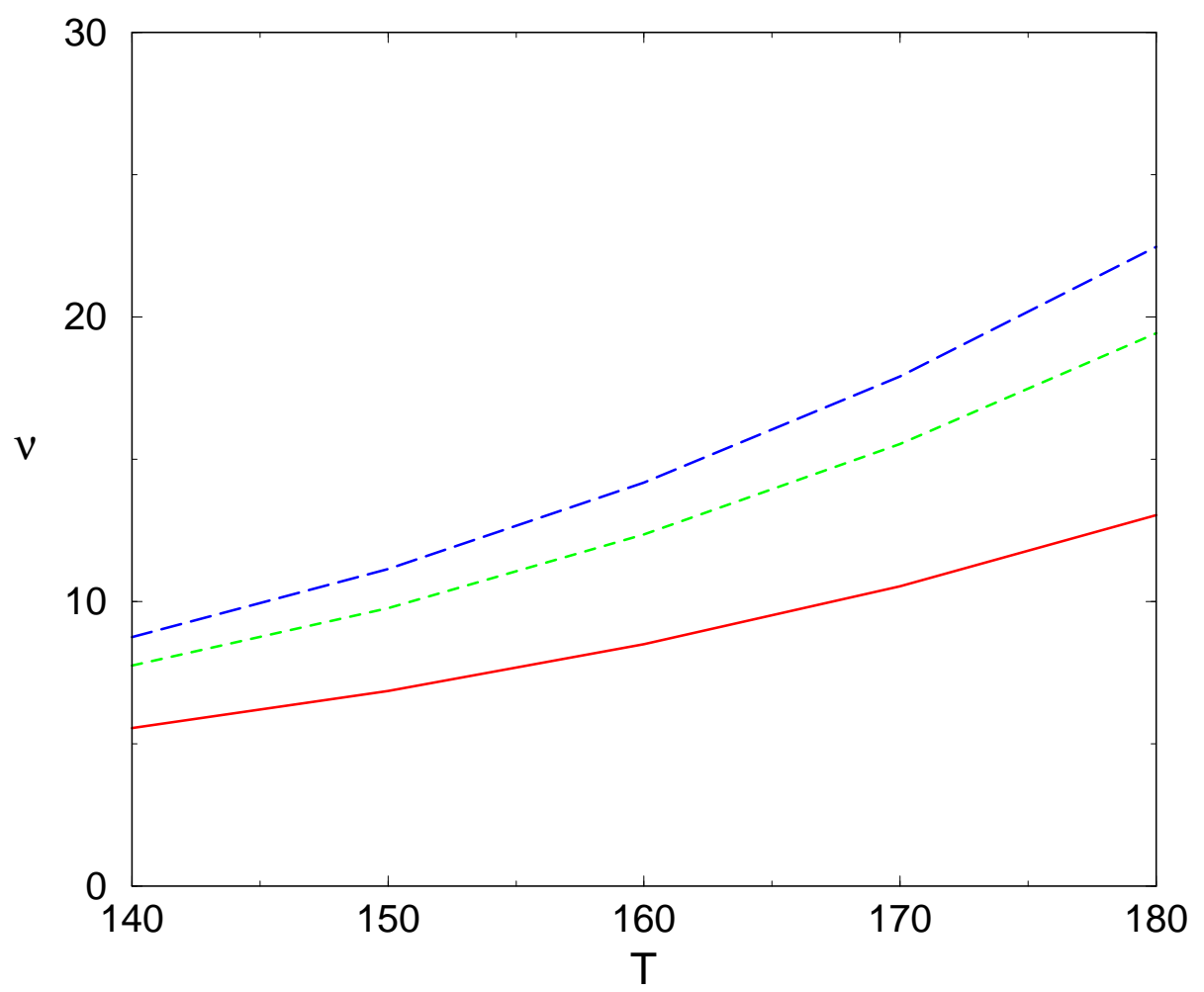

Figure 1: The effective number of degrees of freedom of a hadronic resonance gas, using the three definitions of $\nu$ provided by Eqs. (1-3): (1) long-dashed line (blue), (2) short-dashed line (green), and (3) solid line (red). The solid curve moves by about $5 \%$ depending on whether we use all established resonances in the particle data book or only those commonly included in the chemical freezeout analysis. This can be considered an estimate of the theoretical uncertainty in the curve.

Lattice QCD calculations indicate that throughout the temperature range $2 T_{c}<T<$ $5 T_{c}, \nu(T)$ is between $70 \%$ and $80 \%$ of that for an ideal quark-gluon plasma 画, meaning that $33 \lesssim \nu \lesssim 38$. At lower temperatures, closer to the crossover, the value of $\nu$ extracted from lattice QCD calculations is more significantly different for different definitions of $\nu$, because just above the crossover the pressure deviates more from its ideal gas value than the energy density does. At $T=1.5 T_{c}$, for example, with the definition (1) lattice calculations [4] yield $\nu \approx 37$, whereas with our definition (3) they yield $\nu \approx 27$.

There is an additional benefit to defining $\nu$ via (3) as we do, over and above making the quantity observable in practice. In order to be convincing, a future experimental measurement of $\nu$ that agreed with what lattice QCD predicts for the quark-gluon plasma would need to discriminate between this prediction and that of a hadronic resonance gas with $T \lesssim T_{c}$. We see from Fig. 1 that, with our definition of $\nu$, this requires excluding $\nu \approx 10$. The three different definitions of $\nu$ all agree for a gas of noninteracting massless particles, but massive particles - as in a resonance gas - contribute somewhat less to $s / T^{3}$ than they do to $\varepsilon / T^{4}$, and hence less still to $s^{4} / \varepsilon^{3}$. This makes our definition of $\nu$ advantageous.

Before turning to the challenges associated with measuring $s$ and $\varepsilon$, we note that the 
value of $\nu$ defined from them via (3) can only be compared to QCD thermodynamics if there is independent evidence that the matter under study is in (approximate) local thermal equilibrium. At RHIC, such evidence is believed to be provided by the agreement of the elliptic flow measured in noncentral collisions with hydrodynamic model predictions [1]. Such predictions are based on the assumption that the matter behaves like a fluid in local thermal equilibrium, with arbitrarily short mean free paths and correspondingly strong interactions. The fact that the data show as much elliptic flow as predicted indicates that this assumption must already be valid soon after the collision, early enough that the azimuthal spatial anisotropy due to the nonzero impact parameter has not had time to be significantly reduced via free streaming of weakly interacting quasiparticles. Quantitatively, it is estimated that local thermal equilibrium and the onset of hydrodynamic behavior must occur by a time $t_{0} \sim 0.6-1.0 \mathrm{fm} / \mathrm{c}[7] .{ }^{1}$ We shall take $t_{0}=1.0 \mathrm{fm} / \mathrm{c}$ in the following. In the remainder of this paper, we shall discuss how $s\left(t_{0}\right)$ and $\varepsilon\left(t_{0}\right)$ may be estimated, using present and near-future data.

The estimate of $s\left(t_{0}\right)$ relies on the fact that the entropy of an ideal fluid is conserved during its hydrodynamical evolution. The value of $s\left(t_{0}\right)$ can therefore be deduced from the value of $d S / d y$ at chemical freezeout, upon assuming that the fluid remains in local thermal equilibrium between the time $t_{0}$ when this condition is established and the time at which chemical freezeout occurs. Note that we shall need to know the volume of the system at time $t_{0}$, which is early enough that little transverse expansion has taken place making this easy to estimate, but we shall not need to know the volume of the system at freezeout. Indeed, we do not need to know anything about the system at freezeout except its entropy. In an isentropic expansion, it is entropy that is conserved, regardless of how entropy density evolves.

There are, in principle, two ways of estimating $d S / d y$. One approach uses an analysis of the composition of the fireball at chemical freezeout to derive the entropy per hadron, which can then be used to relate the measured charge particle multiplicity $d N_{\mathrm{ch}} / d y$ to the entropy $d S / d y$, assuming full thermal phase space occupation at the freezeout time. The other approach 9] uses the measured multiplicities of stable hadrons together with experimental data on Hanbury-Brown-Twiss (HBT) two-particle interferometry to estimate the full phase space distributions $f_{i}(\vec{r}, \vec{p})$ at kinetic freezeout, and from them the entropy

$$
S=\sum_{i} \int \frac{d^{3} r d^{3} p}{(2 \pi)^{3}}\left[-f_{i} \ln f_{i} \pm\left(1 \pm f_{i}\right) \ln \left(1 \pm f_{i}\right)\right]
$$

where the upper (lower) sign holds for bosons (fermions).

We begin with a review of the results of the kinetic freezeout analysis. Pal and Pratt 9] have used final state multiplicities and "radius" parameters deduced from a HBT correlation analysis to determine $d S / d y$ in data from $\sqrt{s}=130 A \mathrm{GeV} \mathrm{Au}+\mathrm{Au}$ collisions. For collisions in a $11 \%$ centrality window, Pal and Pratt find $d S / d y \approx 4450$ with an estimated systematic error of \pm 400 . Two effects may affect the reliability of this estimate. First, the

\footnotetext{
${ }^{1}$ Recent work suggests that this rapid approach to local thermal equilibrium may occur via plasma instabilities, not collisions [8]. We caution that if thermal but not chemical equilibrium has been achieved at $t_{0}$, then $\nu\left(t_{0}\right)$ may be lower than the value calculated in lattice QCD.
} 
conventional extraction of HBT radius parameters is based on the assumption that hadrons originate from a source that is Gaussian in both position and momentum space. This is not a good assumption for a rapidly expanding source, and the measured HBT radius parameters do not actually serve as estimates of the radii of the fireball at freezeout. Hence, a more direct measurement of the phase space distributions themselves, without introducing a parametrization in terms of "radii", would be of value. And, second, the entropy of the hadronic gas must increase as it cools after chemical freezeout but before kinetic freezeout. As the mean free path becomes larger and eventually approaches the size of the system at kinetic freezeout, the viscosity of the hadron gas grows, ideal hydrodynamics ceases to be a good approximation, and entropy is produced. Furthermore, after chemical freezeout more resonances decay than are produced, again increasing the entropy. Hence, the entropy at chemical freezeout, which is what is of interest to us, must be smaller than that estimated at kinetic freezeout.

Next, we consider the analysis based on the inferred chemical freezeout multiplicities of hadrons, which has the potential to extract the entropy at chemical freezeout directly. The ratios of hadron multiplicities in RHIC collisions with $\sqrt{s}=200 \mathrm{AGeV}$ are quite well described by the assumption that chemical freezeout occurs at $T_{\text {ch }}=170 \pm 10 \mathrm{MeV}$ from an equilibrated hadron gas at this temperature [10]. An ideal gas of all established meson and baryon resonances at $T=170 \mathrm{MeV}$ has an entropy of about 7.25 per hadron [11, 12], which can be compared to the value of 3.6 for an ideal gas of massless pions. After all the resonances decay the multiplicity of charged hadrons in the final state is 1.04 per hadron in the equilibrated hadron gas [11]. In the $6 \%$ most central collisions with $\sqrt{s}=200 A \mathrm{GeV}$, the multiplicity of charged particles in the final state is $d N_{\mathrm{ch}} / d \eta=665 \pm 26$ at midrapidity [13], corresponding to a $d N_{\mathrm{ch}} / d y$ that is about $10 \%$ larger [14]. Putting the pieces together, we estimate $d S / d y=(665 \times 1.1 \times 7.25) / 1.04 \approx 5100$ at chemical freezeout at midrapidity in RHIC collisions with $\sqrt{s}=200 \mathrm{AGeV}$. There is a $4 \%$ uncertainty in this estimate coming from that in the experimental measurement of $d N_{\mathrm{ch}} / d \eta$. The largest theoretical uncertainty is that in the factor $S / N=7.25$. Changing the chemical freezeout temperature by $\pm 10 \mathrm{MeV}$ changes $S / N$ by $\pm 3 \%$. To get a sense of the other uncertainties in $S / N$, we recalculated it using abundances obtained including the widths of states, and found that $S / N$ increased to 7.58. There are still further sources of theoretical uncertainty that are harder to estimate like, for example, that due to our neglect of resonances not found in the particle data book. We do not think this is a large effect, because reducing the number of resonances that we include does not have a large effect. We estimate $S / N=7.25 \pm 6 \%$, and expect that a more systematic analysis could reduce this uncertainty by a factor of two. Adding the theoretical and experimental uncertainties in quadrature and rounding upwards yields $d S / d y=5100 \pm 400$.

We can also compare our estimate of $d S / d y$ at chemical freezeout to that obtained by Pal and Pratt at kinetic freezeout. Applying our argument to the $11 \%$ most central collisions with $\sqrt{s}=130 \mathrm{AGeV}$, for which $d N_{\mathrm{ch}} / d \eta=526 \pm 20$ [13], yields the estimate $d S / d y=4035 \pm 300$. As expected, the central value of this estimate is below that obtained by $\mathrm{Pal}$ and Pratt, but the difference is within error bars. This indicates that the entropy release between chemical and kinetic freezeout is not dramatic. 
To summarize, the best estimates available at present suggest that $d S / d y \approx 5100 \pm 400$ at chemical freezeout at mid-rapidity in central RHIC collisions with $\sqrt{s}=200 \mathrm{AGeV}$. It seems to us that a more careful theoretical analysis can reduce the theoretical uncertainty by a factor of two, which would yield a measurement of $d S / d y$ with $5 \%$ errors.

To the extent that the expansion between $t_{0}=1 \mathrm{fm} / \mathrm{c}$ and chemical freezeout is isentropic, the argument first proposed by Bjorken [15] can be used to turn $d S / d y$ into a lower bound on $s\left(t_{0}\right)$. The PHOBOS version of this argument [14] can be phrased as follows. The charged particle multiplicity is reasonably independent of the rapidity $y$ within the range $|y|<1$, and all this entropy $(S=2 d S / d y \approx 10200 \pm 800)$ must have come from within a volume of size $2 t_{0} \pi R^{2}$ at time $t_{0}$. Taking $R=7 \mathrm{fm}$, this yields $s\left(t_{0}\right) \geq 33 \pm 3 \mathrm{fm}^{-3}$. The entropy density at $t_{0}$ is somewhat greater than this, because of the contribution from particles outside $|y|<1$.

In order to obtain a lower bound on $\nu\left(t_{0}\right)$ using (3), it is important to have a lower bound on $s\left(t_{0}\right)$, as the Bjorken argument provides upon assuming isentropic expansion. It is crucial, however, to test this assumption. That is, it is crucial to rule out a significant increase of the entropy between equilibration at time $t_{0}$ and chemical freezeout. Entropy production during the hydrodynamic expansion requires some specific mechanism such as a strong first order phase transition which can drive the matter away from local thermal equilibrium. Lattice QCD calculations indicate that the transition is a crossover, with hadronization occuring continuously, but it would be desirable to have experimental confirmation of the absence of a strong first order phase transition, in order to complete the justification of our use of $d S / d y$ at chemical freezeout to obtain a lower bound on $s\left(t_{0}\right)$. A strong first order phase transition would lead to large (and possibly non-Gaussian) eventby-event fluctuations at low $p_{T}$ [16]. There is no evidence for such fluctuations in current data [17], but given the importance of this issue a more stringent investigation of low- $p_{T}$ event-by-event fluctuations is called for, looking at the fluctuations of several observables and focusing on $p_{T}$ significantly smaller than the mean.

Just as it is important to have a lower bound on $s\left(t_{0}\right)$, if we wish to obtain a lower bound on $\nu$ we need an upper bound on $\varepsilon\left(t_{0}\right)$. The analogous "Bjorken argument", applied to $d E_{T} / d y$ (where $E_{T}$ is the total transverse energy of the hadrons in the final state) only yields a lower bound on $\varepsilon\left(t_{0}\right)$ because the longitudinal expansion subsequent to $t_{0}$ reduces $d E_{T} / d y$. For this reason, we cannot use $d E_{T} / d y$ for our purposes. Putting this another way, Bjorken arguments applied to $d N / d y$ and $d E_{T} / d y$ yield lower bounds on both $s\left(t_{0}\right)$ and $\varepsilon\left(t_{0}\right)$, and so by themselves these arguments give no constraint on $\nu$.

The analysis of jet quenching data has the potential to yield an upper bound on the energy density at early times, as we now discuss. High energy partons traversing strongly interacting matter lose energy mainly by gluon radiation after interactions with colored constituents of the medium. The theory of this mechanism is well developed within the framework of perturbative QCD [18, 19, 20, 21, 22, 23]. In the multiple soft scattering limit, the effect of the medium is encoded in the parameter

$$
\hat{q}=\rho \int q^{2} d q^{2}\left(d \sigma / d q^{2}\right)
$$


where $\rho$ is the density of scattering centers in the medium, $q^{2}$ denotes the momentum transfer in scattering, and $d \sigma / d q^{2}$ is the differential cross section for scattering of the hard parton on a single center. Coherence effects suppress the emission of gluons with energies above $\omega_{c}=\hat{q} L^{2} / 2$, where $L$ denotes the length of material traversed. The mean energy lost by the parton is given by $\Delta E \approx\left(3 \alpha_{s} C_{R} / \pi\right) \omega_{c}$, where $C_{R}$ is the Casimir operator for the color representation of the parton. Similar results are obtained in the opposite (low opacity) limit, when the interaction with the medium is dominated by a single or few scatterings [22]. In these analyses, the properties of the matter being probed arise only in the transport coefficient $\hat{q}$. In this sense, jet quenching can be thought of as "measuring q."

The relationship between $\hat{q}$ and the energy density $\varepsilon$ has not been determined in general, but it is known for the limiting cases of cold nuclear matter [20], an ideal pion gas, and an ideal weakly-interacting quark-gluon plasma [24]. When a high energy parton penetrates strongly interacting matter, it resolves the partonic constituents of the medium. In the case of cold nuclear matter, it mainly interacts with the gluon component of the nucleons, and the parameter $\hat{q}$ can be expressed in terms of the gluon distribution in the nucleon [20]. For a medium such as a weakly interacting quark-gluon plasma, in which all partons are deconfined, each quark and gluon contributes to the density of scatterers independently, and $\hat{q}$ is directly given by Eq. (可) in terms of the gluon density and perturbatively screened parton-parton cross section. The resulting energy loss in a thermal quark-gluon plasma has been calculated by Baier as function of the energy density of the plasma [24]. Remarkably, when the energy loss coefficient is calculated for a thermal gas of pions, one finds the same value of $\hat{q}$ as for a thermal quark-gluon plasma with the same energy density. With the benefit of hindsight, this is not entirely surprising, although the precision of the agreement may be coincidental. About half of the momentum of a fast moving hadron is carried by gluons, and roughly half of the energy density of a weakly interacting quark-gluon plasma is contained in gluons. Normalized to the energy density of the medium, a fast moving parton can therefore be expected to encounter roughly the same number of gluons on which it can scatter.

It is presumably naive to think that a measurement of $\hat{q}$ is a measurement of the energy density $\varepsilon$, as the comparison between Baier's results for energy loss in a weakly interacting pion gas and in a weakly interacting quarki-gluon plasma would suggest. Certainly, the relationship between the energy loss of a hard parton traversing a medium and the energy density of the medium requires further elucidation and generalization. The perturbative expression for the radiative energy loss of an energetic parton, which is only known to leading order in the strong coupling $\alpha_{s}$, may get substantial corrections at higher order. A calculation of next-to-leading order corrections to the energy loss in perturbative QCD would be desirable. Although the energy of the penetrating parton provides a large scale, the momentum transfer of the scattering in the medium provides a second, much lower energy scale, which could enter into the NLO corrections. Also, the quantity $\hat{q}$ which provides the link between the observable $\Delta E$ and the energy density $\varepsilon$ is defined only in the context of the perturbative analysis. Since $\varepsilon$ itself is nonperturbatively well-defined, it would ultimately be desirable to understand the relation between parton energy loss 
observables and $\varepsilon$ directly, possibly involving other nonperturbatively defined properties of the medium being probed by the hard parton. Keeping these caveats in mind, we nevertheless expect that the qualitative lessons encoded in Baier's results will survive in a more rigorous treatment.

Jet quenching by itself can never provide a measure of $\nu$. It cannot differentiate between a weakly interacting quark-gluon plasma and a system whose gluon content is the same, but which has much less entropy density because the gluons are bound within hadrons and hence do not directly contribute to the entropy. In a hadron gas, the entropy is (roughly) a count of the hadrons, which are the thermodynamically independent degrees of freedom, whereas a hard parton "sees" the gluons within each hadron. A weakly interacting quark-gluon plasma and a hypothetical weakly interacting pion gas with the same energy density are equally effective at quenching jets, according to Baier's perturbative analysis, but the pion gas has a much lower entropy density and hence a lower $\nu$. Turning it around, if we imagine a hadron gas and a quark-gluon plasma with the same entropy density, the quark-gluon plasma has the smaller energy density, the smaller density of gluonic scatterers (only one per entropically active degree of freedom), and hence the smaller energy loss by a factor that scales like $\nu^{1 / 3}$. The details of this calculation will differ depending on the precise nature of the composition of the medium, but the principle is general: Any hadronic medium will contain several gluons per hadron, but only the entire hadrons will contribute to the entropy. For a deconfined medium, on the other hand, each gluon contributes to the entropy individually, implying a smaller number of gluons (per unit of entropy) capable of scattering an energetic parton. ${ }^{2}$

Given a lower bound on $s\left(t_{0}\right)$, in order to obtain a lower bound on $\nu$, and hence perhaps a demonstration of deconfinement, we will need to use jet quenching data to obtain an upper bound on $\varepsilon\left(t_{0}\right)$. Further theoretical work is required before this can be attempted quantitatively. Even if the relation between jet quenching data and energy density were in hand, current data may not be sufficient to provide an upper bound. The fact that the observed jet quenching in the most central collisions is quite large (the suppression ratio $R_{A A} \ll 1$ ) means that it may be difficult to derive an upper bound on $\hat{q}$ from these data [27]. The observed strong suppression implies that the observed hadrons are the leading partons from jets originating near the surface of the matter [1], as is also indicated by the absence (within current error bars) of any high- $p_{T}$ hadrons from the away-side jet in the most central collisions [28]. Indeed, the currently ongoing careful study of the material

\footnotetext{
${ }^{2}$ As an aside, we should note that one proposed way of understanding the lattice QCD result that $\nu$ in the quark-gluon plasma is somewhat less than that for an ideal quark-gluon plasma invokes the possibility that, in the energy density range relevant to the RHIC experiments, matter in the deconfined phase may contain colored bound states of quarks and gluons 25]. This reduces the entropy density somewhat without reducing the density of gluonic scatterers in the medium. This is another illustration of our logic, but it is not directly relevant to the strategy for measuring $\nu$ that we propose. Any bound on $\nu$ extracted from data can be compared directly to lattice QCD calculations, regardless of the mechanism by which the strong interactions create the deviation of $\nu$ from its ideal quark-gluon plasma value. Note also that colored bound state formation among quarks and gluons introduces a new contribution to the energy loss, that coming from ionization of the bound states [26]. Neglecting this contribution in a future analysis of jet quenching would mean that the inferred energy density is greater than the true energy density, and thus would not interfere with the goal of obtaining an upper bound on the energy density.
} 
recoiling against a jet originating from near the surface of the matter, for example via dihadron distributions, will provide us with data on new jet quenching observables and could teach us more about the matter produced in central collisions. However, since an upper bound on the energy density must take the form of a statement that if $\varepsilon$ were larger more suppression would have been seen, jet quenching data from noncentral collisions may be a more powerful source of information. Indeed, the away-side jet does not fully disappear in noncentral collisions, especially when the jets only have to fight their way through the hot matter in the narrow direction [28], giving hope that a quantitative upper limit for the value of $\hat{q}$ may not be far away. Data at higher $p_{T}$ and with higher statistics is required, and should come from the Run-4 data set now being analyzed.

Although the above paragraph is our true conclusion, we would be remiss to end without attempting to "plug in numbers just for fun", even absent a reliable upper bound on $\varepsilon\left(t_{0}\right)$. By applying the Bjorken argument to $d E_{T} / d y$, both PHOBOS and PHENIX estimate that $\varepsilon(1 \mathrm{fm} / c))>5 \mathrm{GeV} / \mathrm{fm}^{-3}[1]$. This lower bound on the energy density is of considerable interest in and of itself, even though by itself it cannot be used to constrain $\nu$. Indeed, if we take the lattice QCD calculation of $\nu(T)$ as a given, the experimental lower bound on $\varepsilon$ tells us that at $t=1 \mathrm{fm} / c$ the matter produced at RHIC is a quark-gluon plasma with a temperature $T>1.4 T_{c}$, well above the crossover. In other words, using (1), a lower bound on $\varepsilon$, and the lattice calculation of $\nu$, we obtain a lower bound on $T$ and a "demonstration" of deconfinement, albeit one that is unsatisfying because it uses the lattice calculation of $\nu$ rather than testing it. In order to measure $\nu$ and (presumably) demonstrate deconfinement, what is required is a lower bound on $s\left(t_{0}\right)$ and an upper bound on $\varepsilon\left(t_{0}\right)$. If we adopt the conclusion from above that $s\left(t_{0}\right)>33 \pm 3 \mathrm{fm}^{-3}$ and, absent a reliable upper bound, suppose that $\varepsilon(1 \mathrm{fm} / c)$ is given by 5,7 or $9 \mathrm{GeV} / \mathrm{fm}^{-3}$, we would conclude that $\nu>71 \pm 22, \nu>26 \pm 8$ or $\nu>12 \pm 4$, respectively. A $5 \%$ determination of $s\left(t_{0}\right)$ would reduce these error bars significantly, which motivates the theoretical effort needed to accomplish this goal. Another direction in which theoretical effort is needed is the modelling of the consequences of the variation of $s$ and $\varepsilon$ across the transverse extent of the collision region, something we have not considered here. A stringent experimental investigation of low- $p_{T}$ event-by-event fluctuations is also required, in order to augment current theoretical evidence with experimental evidence against a strong first order phase transition, whose attendant entropy production would complicate the extraction of $\nu$ that we propose. And, most important, these numbers make very clear the importance of further analysis of jet quenching theory and data with the goal of setting a reliable upper bound on $\varepsilon\left(t_{0}\right)$. If there were experimental evidence that $\varepsilon(1 \mathrm{fm} / c)<7 \mathrm{GeV} / \mathrm{fm}^{-3}$, this would be evidence for deconfinement.

Looking further ahead, if RHIC data can provide interesting limits on the value of $\nu$, data from heavy ion collisions at the LHC should do even better. And, QCD predicts that if $\nu$ is above the crossover at RHIC, its value will not increase significantly at the LHC. It is a greater challenge to devise a way of measuring $\nu$ at lower energy densities using lower energy heavy ion collisions, where jet quenching is not observable. 
Acknowledgments: We thank R. Baier, W. Busza, U. Heinz, P. Jacobs, T. Renk, G. Roland, X. Wang, U. Wiedemann and W. Zajc for helpful discussions. We also thank the organizers and participants of the RIKEN-BNL workshop "New Discoveries at RHIC", which provided the stimulus for this work, and the organizers and participants of the "Hard Probes 2004" conference in Ericeira, Portugal, where this work was discussed. This research was supported in part by the U.S. Department of Energy (D.O.E.) under grant \#DE-FG02-96ER40945 and cooperative research agreement \#DF-FC02-94ER40818.

\section{References}

[1] I. Arsene et al. [BRAHMS Collaboration], arXiv: nucl-ex/0410020; K. Adcox et al. [PHENIX Collaboration], arXiv:nucl-ex/0410003; B. B. Back et al. [PHOBOS Collaboration], arXiv: nucl-ex/0410022; J. Adams et al. [STAR Collaboration], arXiv: nucl-ex/0501009.

[2] R. J. Fries, B. Müller, C. Nonaka and S. A. Bass, Phys. Rev. Lett. 90, 202303 (2003) [arXiv:nucl-th/0301087]; Phys. Rev. C 68, 044902 (2003) [arXiv:nucl-th/0306027]; V. Greco, C. M. Ko and P. Levai, Phys. Rev. Lett. 90, 202302 (2003) [arXiv:nucl-th/0301093]; Phys. Rev. C 68, 034904 (2003) [arXiv:nucl-th/0305024]; D. Molnar and S. A. Voloshin, Phys. Rev. Lett. 91, 092301 (2003) [arXiv:nucl-th/0302014]; R. C. Hwa and C. B. Yang, Phys. Rev. C 70, 024905 (2004) [arXiv:nucl-th/0401001]; R. J. Fries, J. Phys. G 30, S853 (2004) [arXiv:nucl-th/0403036].

[3] For reviews, see K. Rajagopal, arXiv:hep-ph/9504310; Acta Phys. Polon. B 31, 3021 (2000) [arXiv:hep-ph/0009058].

[4] For reviews, see F. Karsch, Nucl. Phys. A 698, 199 (2002) [arXiv:hep-ph/0103314]; Lect. Notes Phys. 583, 209 (2002) [arXiv:hep-lat/0106019]; P. Petreczky, arXiv:hep-lat/0409139.

[5] F. Karsch, J. Phys. G 30, S887 (2004) [arXiv:hep-lat/0403016]. M. Asakawa and T. Hatsuda, Nucl. Phys. A 721, 869 (2003).

[6] F. Karsch and H. W. Wyld, Phys. Rev. D 35, 2518 (1987); G. Aarts and J. M. Martinez Resco, JHEP 0204, 053 (2002) [arXiv:hep-ph/0203177]; S. Gupta, Phys. Lett. B 597, 57 (2004) [arXiv:hep-lat/0301006]; A. Nakamura and S. Sakai, arXiv:hep-lat/0406009.

[7] U. W. Heinz and P. F. Kolb, Nucl. Phys. A 702, 269 (2002) [arXiv:hep-ph/0111075].

[8] P. Romatschke and M. Strickland, Phys. Rev. D 68, 036004 (2004) [arXiv:hep-ph/0304092]; P. Arnold, J. Lenaghan and G. D. Moore, JHEP 0308, 002 (2003) [arXiv:hep-ph/0307325]; P. Arnold, J. Lenaghan, G. D. Moore and L. G. Yaffe, arXiv:nucl-th/0409068; P. Romatschke and M. Strickland, arXiv:hep-ph/0408314.

[9] S. Pal and S. Pratt, Phys. Lett. B 578, 310 (2004) [arXiv:nucl-th/0308077].

[10] P. Braun-Munzinger, J. Stachel and C. Wetterich, Phys. Lett. B 596, 61 (2004) [arXiv:nucl-th/0311005]; M. Kaneta and N. Xu, arXiv:nucl-th/0405068; for a review, see P. Braun-Munzinger, K. Redlich and J. Stachel, arXiv:nucl-th/0304013.

[11] C. Nonaka, S. A. Bass, B. Müller, and M. Asakawa, arXiv:nucl-th/0501028.

[12] For earlier work in this direction, see J. Sollfrank and U. W. Heinz, Phys. Lett. B 289, 132 (1992).

[13] B. B. Back et al. [PHOBOS Collaboration] Phys. Rev. C 65, 061901 (2002) [arXiv:nucl-ex/0201005]. 
[14] B. B. Back et al. in Ref. [1], and references therein.

[15] J. D. Bjorken, Phys. Rev. D 27, 140 (1983).

[16] H. Heiselberg and A. D. Jackson, arXiv:nucl-th/9809013; Phys. Rev. C 63, 064904 (2001) [arXiv:nucl-th/0006021]; I. N. Mishustin, Phys. Rev. Lett. 82, 4779 (1999) [arXiv:hep-ph/9811307].

[17] K. Adcox et al. [PHENIX Collaboration], Phys. Rev. C 66, 024901 (2002) [arXiv:nucl-ex/0203015]; J. Adams et al. [STAR Collaboration], Phys. Rev. C 68, 044905 (2003) [arXiv:nucl-ex/0307007]; arXiv:nucl-ex/0308033; S. S. Adler et al. [PHENIX Collaboration], Phys. Rev. Lett. 93, 092301 (2004) [arXiv:nucl-ex/0310005].

[18] X. N. Wang, M. Gyulassy and M. Plumer, Phys. Rev. D 51, 3436 (1995) [arXiv:hep-ph/9408344].

[19] R. Baier, Y. L. Dokshitzer, A. H. Mueller, S. Peigne and D. Schiff, Nucl. Phys. B 483, 291 (1997) [arXiv:hep-ph/9607355].

[20] R. Baier, Y. L. Dokshitzer, A. H. Mueller, S. Peigne and D. Schiff, Nucl. Phys. B 484, 265 (1997) [arXiv:hep-ph/9608322].

[21] B. G. Zakharov, JETP Lett. 63, 952 (1996) [arXiv:hep-ph/9607440]; R. Baier, Y. L. Dokshitzer, A. H. Mueller and D. Schiff, Phys. Rev. C 58, 1706 (1998) [arXiv:hep-ph/9803473]; X. N. Wang and X. F. Guo, Nucl. Phys. A 696, 788 (2001) [arXiv:hep-ph/0102230]; E. Wang and X. N. Wang, Phys. Rev. Lett. 89, 162301 (2002) [arXiv:hep-ph/0202105].

[22] M. Gyulassy, P. Levai and I. Vitev, Phys. Rev. Lett. 85, 5535 (2000) [arXiv:nucl-th/0005032]; Nucl. Phys. B 594, 371 (2001) [arXiv:nucl-th/0006010]; U. A. Wiedemann, Nucl. Phys. B 588, 303 (2000) [arXiv:hep-ph/0005129].

[23] For reviews, see R. Baier, D. Schiff and B. G. Zakharov, Ann. Rev. Nucl. Part. Sci. 50, 37 (2000) [arXiv:hep-ph/0002198]; A. Kovner and U. A. Wiedemann, in Quark Gluon Plasma 3, eds. R. C. Hwa and X. N. Wang (World Scientific, Singapore, 2004) [arXiv:hep-ph/0304151]; A. Accardi et al., arXiv:hep-ph/0310274.

[24] R. Baier, Nucl. Phys. A 715, 209 (2003) [arXiv:hep-ph/0209038].

[25] E. V. Shuryak and I. Zahed, Phys. Rev. C 70, 021902 (2004) [arXiv:hep-ph/0307267]; Phys. Rev. D 70, 054507 (2004) [arXiv:hep-ph/0403127].

[26] E. V. Shuryak and I. Zahed, arXiv:hep-ph/0406100.

[27] K. J. Eskola, H. Honkanen, C. A. Salgado and U. A. Wiedemann, Nucl. Phys. A 747, 511 (2005) [arXiv:hep-ph/0406319].

[28] C. Adler et al. [STAR Collaboration], Phys. Rev. Lett. 90, 082302 (2003); J. Adams et al. [STAR Collaboration], Phys. Rev. Lett. 91, 072304 (2003); Phys. Rev. Lett. 93, 252301 (2004) [arXiv:nucl-ex/0407007]; J. Adams et al. in Ref. [1]. 